\title{
Fegebank, Barbara
}

\section{Der Wunschverbraucher heißt Homo oecologicus}

Haushalt in Bildung \& Forschung 5 (2016) 3, S. 19-34

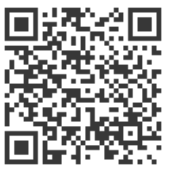

Quellenangabe/ Reference:

Fegebank, Barbara: Der Wunschverbraucher heißt Homo oecologicus - In: Haushalt in Bildung \& Forschung 5 (2016) 3, S. 19-34 - URN: urn:nbn:de:0111-pedocs-203313 - DOI: 10.25656/01:20331

https://nbn-resolving.org/urn:nbn:de:0111-pedocs-203313

https://doi.org/10.25656/01:20331

in Kooperation mit / in cooperation with:

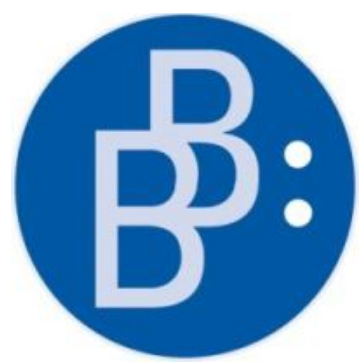

https://www.budrich.de

\section{Nutzungsbedingungen}

Gewährt wird ein nicht exklusives, nicht übertragbares, persönliches und beschränktes Recht auf Nutzung dieses Dokuments. Dieses Dokument ist ausschließlich für den persönlichen, nicht-kommerziellen Gebrauch bestimmt. Die Nutzung stellt keine Übertragung des Eigentumsrechts an diesem Dokument dar und gilt vorbehaltlich der folgenden Einschränkungen: Auf sämtlichen Kopien dieses Dokuments müssen alle Urheberrechtshinweise und sonstigen Hinweise auf gesetzlichen Schutz beibehalten werden. Sie dürfen dieses Dokument nicht in irgendeiner Weise abändern, noch dürfen Sie dieses Dokument für öffentliche oder kommerzielle Zwecke vervielfältigen, öffentlich ausstellen, aufführen, vertreiben oder anderweitig nutzen.

Mit der Verwendung dieses Dokuments erkennen Sie die Nutzungsbedingungen an.

\section{Terms of use}

We grant a non-exclusive, non-transferable, individual and limited right to using this document.

This document is solely intended for your personal, non-commercial use. Use of this document does not include any transfer of property rights and it is conditional to the following limitations: All of the copies of this documents must retain all copyright information and other information regarding legal protection. You are not allowed to alter this document in any way, to copy it for public or commercial purposes, to exhibit the document in public, to perform, distribute or otherwise use the document in public.

By using this particular document, you accept the above-stated conditions of use.

\section{Kontakt / Contact:}

\section{peDOCS}

DIPF | Leibniz-Institut für Bildungsforschung und Bildungsinformation Informationszentrum (IZ) Bildung

E-Mail: pedocs@dipf.de

Internet: www.pedocs.de

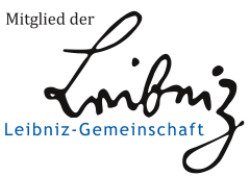




\section{Jahrgang} Heft 3 2016 व

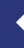

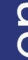
0

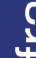

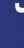

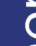

$\frac{\pi}{\mathrm{C}}$

(1)

항

t)

$\bullet$

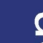

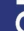

8

$\frac{1}{c}$

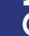

$\xi$

政

$\stackrel{0}{N}$

$\diamond$

$\pm$

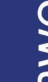

$\underline{\xi}$

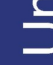

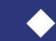

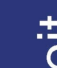

过

ISSN 2193-8806

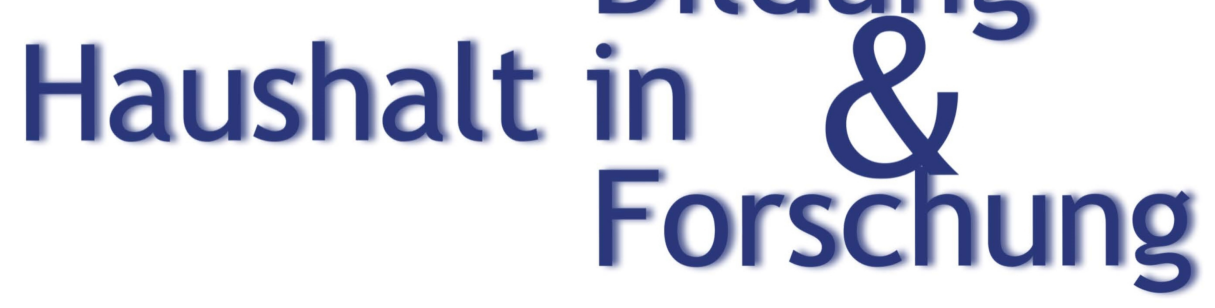

Die ,postmodernen" Konsument/inn/en

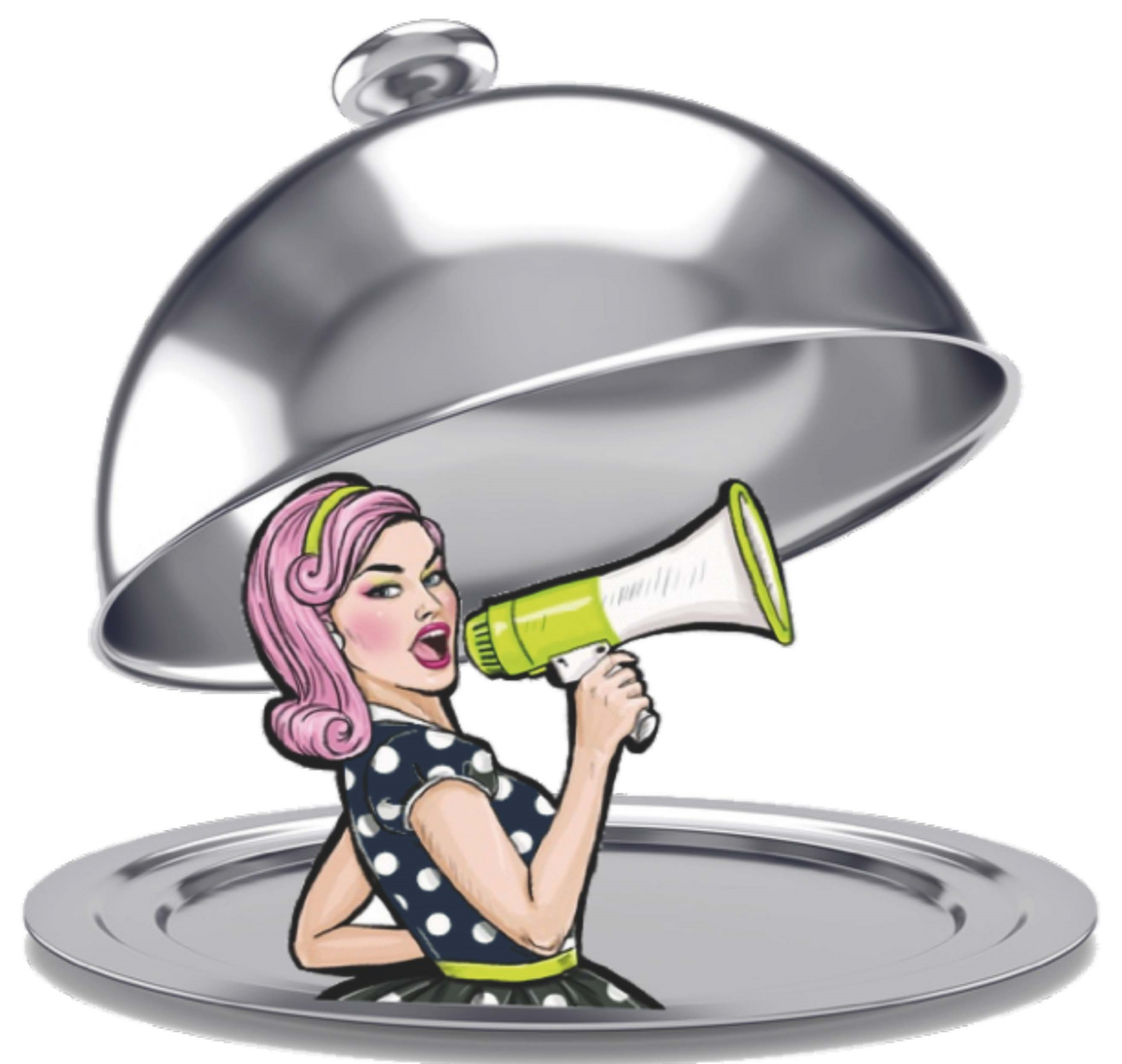

Verlag Barbara Budrich 


\section{Inhaltsverzeichnis}

Werner Brandl

Editorial.

Burkhard Bierhoff

Die Konsumgesellschaft und der postmoderne Konsument 3

Barbara Fegebank

Der Wunschverbraucher heißt Homo oecologicus

Nadine Heiduk

Standortbestimmungen: Reflektierter Konsum zwischen

kollektiven Konsumkrisen und individueller Gestaltungsmacht

Birgit Peuker

Das eklektische Verbraucherverhalten - einen Verbrauchertypus neu denken?

Gabriela Leitner

Postmoderne Bildung für postmoderne Konsument/inn/en 59

Georg Raacke

Einzukaufen ist nicht schwer - Konsument zu sein dagegen sehr 69

Irene Antoni-Komar

Urban Gardening, Food Coops, Community Supported Agriculture:

Transformative Wirtschaftsformen - Konsumpraktiken - Marktbeziehungen.

Christine Brombach

Convenienceprodukte und Essen: Zeitgewinn oder Kosten

für den Bereich der Beköstigung im Privathaushalt?

Werner Brandl

Der Konsum/ent - Anfragen und Anmerkungen

zu Phänomen, Funktion und Option

Thomas Schröder

Ernährungstrends im Kontext von Individualisierung und Identität. 127 
Homo oecologicus |

\section{Barbara Fegebank}

\section{Der Wunschverbraucher heißt Homo oecologicus}

Dies ist eine Behauptung, die es zu begründen gilt und deren Geltungsanspruch bzw. Gültigkeit aufzudecken ist. Die hier dargelegte Argumentationskette dient somit der Beweisführung, bedarf deshalb begrifflicher Klärungen und eines kritischen Blickes in die Vergangenheit der Auseinandersetzung mit dem Verbraucher ${ }^{1}$ und der Leitbilder für das Verbraucherverhalten.

Schlüsselwörter: Bildungspolitik, Leitbilder, Nachhaltigkeit, Verbraucherbildung, Verbraucherpolitik

\section{Der Verbraucher in wissenschaftlicher und politischer Auseinandersetzung}

Es gab eine lange Auseinandersetzung mit dem Konsum, Verbrauch und der Nachfrage - Begriffe, die häufig synonym verwendet werden - in einzelnen Wissenschaften, insbesondere in der Volkswirtschaftslehre, deren Erkenntnisse bis in die jüngste Zeit hineinwirken. Der Verbrauch - noch nicht der Verbraucher - wurde zum wissenschaftlichen Gegenstand als die Volkswirtschaftslehre entstand und die Wirtschaft der Zünfte und Städte ablöste.

Im Verlauf der Geschichte werden zumeist drei große Untersuchungsbereiche in der Volkswirtschaftslehre (VWL), in der auch Verbrauch und Nachfrage Berücksichtigung finden, unterschieden (vgl. Schmölders, 1962).

Während der Zeit des Merkantilismus (ab dem 17. Jhd.) fand eine erste planmäßige Befassung mit volkswirtschaftlichen Problemen, makroökonomischen Problemen, statt und damit wurde auch der Verbrauch in seiner Bedeutung im Wirtschaftskreislauf gesehen. Dies wurde noch deutlicher bei den Physiokraten, die als eigentliche Urheber der Nationalökonomik angesehen werden. Merkantilisten und Physiokraten sahen in dem Konsum einen Gegensatz zur Produktion, der Konsum galt als abhängige Variable der Produktion; er ist das Mittel, die Produkte von Handel, Gewerbe und Landwirtschaft in den Umlauf zu bringen. Dabei wurden die Produkte des einen Marktpartners zur Nachfrage des anderen, die Ausgaben des einen zur Einnahme des anderen. Eine autonome Änderung der Nachfrage wurde als Möglichkeit nicht gesehen; man räumte allenfalls eine Nachfragesteigerung mit einem Bevölkerungszuwachs ein. 


\section{Homo oecologicus}

Die Vertreter der klassischen Schule der Nationalökonomie gingen dann davon aus, dass der Mensch bei der Verfolgung seiner eigennützigen, wirtschaftlichen Ziele dem Gemeinwohl am besten dient, und forderten folglich, dass sich der Staat nicht in die Abläufe der Wirtschaft einmischen sollte. Hier galt der Konsum als Wertvernichtung. Er wurde auch im Zusammenhang mit der Produktion gesehen, jedoch nicht im gesamten Wirtschaftskreislauf, sondern im Marktgeschehen, so dass einmal vom Markt Genommenes keinen Wert mehr hatte.

Schließlich wurden - in der Neoklassik - mikroökonomische Untersuchungen zur Entstehung der Nachfrage durchgeführt, ihre Bestimmungsfaktoren heraus gearbeitet und Erklärungen für das Nachfrageverhalten gesucht. Hiermit begann die ,, subjektive Wertlehre“, die das Motiv der Bedürfnisbefriedigung in ihre Untersuchungen aufnahm, den Konsumenten in den Mittelpunkt rückte. Seine Beurteilung von der Nützlichkeit der Güter war der Maßstab für Wert und Preis der Güter. Bis heute ist jedoch das Problem der ,Subjektivität“ des Nutzens, der nicht quantitativ messbar ist, nicht gelöst.

Mit den Institutionalisten, ein amerikanischer Beitrag zur Wirtschaftstheorie, wurden zum ersten Mal die Wirtschaftssubjekte in den Fokus gerückt. „Das Prinzip, das die Menschen zum Handeln antreibt, das hinter fast jeder einzelnen Lebensäußerung steht, ist das Bedürfnis, sich vor anderen Menschen auszuzeichnen, ,neidvolle Vergleiche“ zu ziehen, wie Veblen sagt, das Verlangen nach Prestige“" (Hasselberg \& Heintz, 1971, S. 8). Mit solchen Erkenntnissen befassten sich fernerhin die Einzelwirtschaftslehren, insbesondere die Betriebswirtschaftslehre, die den Blick von der Funktion des Absatzes zu einer marktorientierten Unternehmensführung richtete und mit den Marketingstrategien, insbesondere der Werbung versuchte, die Verbraucher für sich zu gewinnen.

Im letzten Jahrhundert wurde das wirtschaftliche Handeln auch Gegenstand in Soziologie und Psychologie. So befasste sich George Katona mit der Psychologie des Massenkonsums und die Sozialkritiker Vance Packard und John Kenneth Galbraith mit den „Geheimen Verführern“ bzw. der „Gesellschaft im Überfluß“; es waren Veröffentlichungen aus den USA, die nach dem 2. Weltkrieg dann auch in Deutschland Beachtung fanden. In Deutschland beschäftigte sich der Psychologe Ulrich Beer Ende der 1960er Jahre mit dem wachsenden Konsum, der zu einem pädagogischen Problem zu werden scheint, und er stellt die Fragen: Wird die Jugend mit ihrer neuen, bedeutenden Rolle im Wirtschaftsgefüge fertig? Werden die jungen Menschen von der Konsumgüterwerbung weiterhin in die Konsumschlacht geworfen werden, oder werden sie - durch Erziehung und Bildung vorbereitet - die „gelernten Verbraucher“ von morgen sein? (vgl. Beer, 1967, Umschlag).

In der heutigen Zeit wird der Konsum sowohl makro- als auch mikroökonomisch, aber auch soziologisch, psychologisch, kulturanthropologisch und von Politologen untersucht. Dabei wird Konsum sowohl als Gegenpol zur Produktion als auch als Bedürfnisbefriedigung, aber auch als Prozess und Antriebskraft im Wirt- 


\section{Homo oecologicus |}

schaftsleben aufgefasst. Dennoch ist die Marktbetrachtung dominant geblieben und die wesentlichen Fragen des Konsums sind die Fragen des Verhaltens geworden, eines Verhaltens, das sich seit den 1950er Jahren in West-Deutschland durch den wirtschaftlichen Aufschwung und die stetig steigenden Einkommen gravierend verändert hat. Die Marktgeschehnisse wurden nahezu unüberschaubar, der „König Kunde“, der „souveräne Konsument“ zeigte Schwächen und so weckte der Konsument auch das Interesse der Politik, und die Ökotrophologie mit der Haushaltsund Ernährungswissenschaft, die sich ab 1962 an westdeutschen Universitäten etablierte, machte die Verbraucherlehre zu einem ihrer Schwerpunkte (z. B. Schmucker), was allerdings in der Wissenschaftsgesellschaft nicht wahrgenommen wird (vgl. Solomon et al., S. 38).

Die politische Auseinandersetzung mit dem Verbrauch kann ebenfalls auf eine lange Geschichte zurückblicken, da es schon immer im staatlichen Interesse war, die Versorgung der Bevölkerung zu gewährleisten. Verbraucherpolitik bezeichnet allerdings nicht nur die staatlichen, parteipolitischen, sondern auch verbandlichen Aktivitäten, die dazu dienen, die Marktposition der privaten Endabnehmer und Konsumenten gegenüber den Produzenten, Händlern und Dienstleistungsanbietern zu stärken. Ab 1860 entstanden die ersten Verbrauchervereinigungen, wie die bürgerlich-mittelständische Vereinigung, Arbeiterkonsumvereine und christliche Konsumgenossenschaften. Auch die zu dieser und etwas späterer Zeit gegründeten Frauenvereine waren „verbraucherpolitisch“ aktiv. Während der Weimarer Zeit und des Hitler-Regimes führten die Konsumgenossenschaften und auch viele Vereine eher ein Schattendasein oder wurden gar aufgelöst, so dass man in der Bundesrepublik Deutschland von einer Verbraucherpolitik erst nach dem zweiten Weltkrieg und nach Überwindung der „Hungerzeit“, ab 1950 sprechen kann.

Schmucker erklärte die Etablierung einer Verbraucherpolitik mit der sog. „freien Einkommensspitze“ und der „strukturellen Schwäche des Verbrauchers am Markt" (aus ihren Vorlesungen 1969-1971); in der Öffentlichkeit erfolgte die Sensibilisierung für die Auseinandersetzung mit dem Verbraucher/verhalten u. a. über die Bücher von Galbraith, Veblen und Packard, die insbesondere die „neue“ Konsumgesellschaft kritisierten. Die boomende Wirtschaft, die immer schneller immer mehr Konsumgüter hervorbrachte und einen differenzierten Konsumgüter- schließlich auch Dienstleistungsmarkt entstehen ließ, überforderte die Verbraucher, die im wirtschaftlichen Aufschwung ihr "Mehr" an Einkommen ausgeben wollten und sollten.

Von den 1950er Jahren bis zu den 1970er Jahren kann man verbraucherpolitisch drei Stränge ausmachen, die getragen wurden von Verbänden, Wissenschaftlern und Politikern. Am 30. April 1953 wurde die Arbeitsgemeinschaft der Verbraucherverbände e.V. $(A g V)$ als „Verband der Verbände“ in der Bundesrepublik Deutschland gegründet. Ziel war die Bündelung der verbraucherpolitischen Arbeit zahlreicher Verbände und Steigerung der Bedeutung von Verbraucherpolitik an 


\section{Homo oecologicus}

sich. Mitgliedsverbände der $\mathrm{AgV}$ wurden dann nach und nach der Deutsche Mieterbund, der Frauenring, der Deutsche Hausfrauenbund und viele andere. Am 1. November 2000 ging die $\mathrm{AgV}$ auf im neugegründeten Verbraucherzentrale Bundesverband. 1953 wurde in Berlin auch die erste Verbraucherzentrale gegründet, der weitere (inzwischen in jedem Bundesland) folgten.

Nach und nach wurde durch die Verbandsaktivitäten der Weg geebnet für die staatliche, parteipolitische Auseinandersetzung mit den Verbrauchern, in Westdeutschland zunächst im Bundes- und Landwirtschaftsministerium. Erste schriftliche Zeugnisse, die der Öffentlichkeit zugänglich waren, sind die Berichte der Bundesregierung zur Verbraucherpolitik, die 1971 und 1972 erschienen, und Ziele, Regelungen und Konzepte auswiesen, die z. T. an Aktualität nichts verloren haben. Parallel erfolgte auch eine „neue“ wissenschaftliche Auseinandersetzung mit den Marktgeschehnissen; es wurden Konzeptionen entwickelt/offengelegt, aber auch kritisch die Entwicklung der Verbraucherpolitik verfolgt. Das betraf die Konzeptionen und die Maßnahmen der Verbraucherpolitik, die auch als Handlungsbereiche der Verbraucherpolitik ausgewiesen werden. Dazu gehörten zunächst die Verbraucherinformation und -aufklärung, später die Verbraucherberatung (alles Aufgaben der Verbraucherzentralen und nachgeordneter Beratungsstellen, zuvor schon Aufgaben von Verbänden und inzwischen auch zahlreicher privater Dienstleistungsanbieter). Fernhin wurde der Verbraucherschutz eine bedeutende Maßnahme der Verbraucherpolitik. Der Schutz des Verbrauchers erfolgt im Wesentlichen über Richtlinien und Gesetze, die mittlerweile alle Bereiche des Konsums betreffen.

Mit dem Vertrag von Maastricht (1991) wurde der Verbraucherpolitik der Rang eines eigenen Politikfeldes verliehen, allerdings ist in Deutschland die ministerielle Zuständigkeit lange Zeit Streitpunkt gewesen. War bis vor wenigen Jahren noch das BM für Ernährung und Landwirtschaft für den Verbraucherschutz zuständig, so ist es nun das Justizministerium. In früheren Zeiten war es gar das Wirtschaftsministerium, das (angeblich) die Interessen der Verbraucher vertrat. Geht man die Bundesministerien durch, so gibt es kaum eines, das nicht durch verbraucherpolitische Belange tangiert wird, so dass man sich fragt, warum nicht den ,uralten" Forderungen nach Errichtung eines eigenen Ministeriums nachgekommen worden ist bzw. wird.

Noch offensichtlicher wird dieses Ansinnen, wenn man sich die zeitlich zuletzt eingeführte Maßnahme - die Verbrauchererziehung und -bildung - vor Augen führt.

\section{Verbraucherbildung und -erziehung}

Allerdings ist die Verbraucherbildung - vor allem, wenn sie in (staatlichen) Bildungseinrichtungen erfolgt - von der Bildungspolitik bestimmt. 


\section{Homo oecologicus |}

Während Verbraucherpolitik es sich u. a. durch die Instrumente der Gesetzgebung, durch behördliche Kontrolle und Überwachung zur Aufgabe machen muss, für den Schutz der Verbraucherinnen und Verbraucher zu sorgen, zielt Verbraucherbildung auf deren Aktivierung zu eigenverantwortlichem Handeln. (Müller \& Mackert, 2003, S. 20)

Wenn es im 1. Verbraucherbericht der Bundesregierung heißt, dass Verbraucher Grundkenntnisse haben, „die zum Verständnis wirtschaftlicher, ernährungsphysiologischer und juristischer Tatbestände notwendig sind" und dass mit der Vermittlung dieser Kenntnisse in den Schulen begonnen werden muss (BMWF, 1971, S. 17), dann ist die Verbraucherpolitik auf die Bildungspolitik angewiesen. Es wurden dann auch Anstrengungen durch die Bundesregierung unternommen, mit Hilfe von Arbeitsgruppen, die heterogen besetzt waren und Kontakte zu Verbraucherorganisationen unterhalten sollten, die Verbraucherbildung umzusetzen; auch wurde für Lehrkräfte die Herausgabe von Material zum Verbraucherunterricht angeregt und finanziell gefördert (vgl. BMWF, 1972, S. 34). 1975 äußert sich Scherhorn kritisch zum Verhältnis von Verbraucher- und Bildungspolitik, was umso verständlicher war, da die Verbraucherpolitik dem Politikfeld Wirtschaft zugeordnet war, und bundespolitische Vorgaben auf das föderalistische System im Bildungsbereich stießen. Das Leitbild eines ,mündigen Verbrauchers“ Wissenschaft bereits diskutiert - gehörte in der Bildungspolitik noch nicht zu den Zielvorstellungen (vgl. Scherhorn, 1975, S. 125).

Es tat sich auch die Frage auf, ob Verbraucherbildung in einem eigenständigen Fach gelehrt, oder integrativer Bestandteil bereits bestehender Fächer werden sollte. $\mathrm{Zu}$ einem eigenständigen Fach ist es nicht gekommen, da bereits in der Ökotrophologie und den Schulfächern der „Hauswirtschaft“ (Haushaltslehre) ${ }^{2}$ der Verbraucherlehre im Sinne des wirtschaftlich ausgerichteten haushälterischen Handelns Rechnung getragen wurde. Außerdem wurde parallel die „Arbeitslehrediskussion" geführt, die ihren Anfang bereits in den 1960er Jahren nahm. Arbeitslehre als Hinführung zur Wirtschafts- und Arbeitswelt ging als Fach, Bereich oder Prinzip in die Schulen ein, beinhaltete häufig die Fächer Hauswirtschaft, Technik und Wirtschaftslehre und war somit auch auf das Verbraucherverhalten ausgerichtet. Dabei wird zuweilen zwischen Verbrauchererziehung und Verbraucherbildung unterschieden, wobei diese Unterscheidung für den vorliegenden Sachverhalt keine Rolle spielt, denn vielfach sind die Ziele bzw. Zielperspektiven in Bildung und Erziehung die gleichen.

Zur schulischen Eingliederung der Verbraucherbildung und -erziehung gab es zahlreiche Bemühungen in den einzelnen Bundesländern, zu denen dann erste wertende Bestandsaufnahmen veröffentlicht wurden, in denen z. B. ,immer noch wirtschaftsfeindliches Gebaren“ (Bick \& Meiser, 1977, S. 5) kritisiert wurde. Außerdem hieß es, dass der Verbraucher zu Handlungsfähigkeit erzogen werden muss mit dem Erziehungsziel des kritisch motivierten und rational urteilenden Wirt- 


\section{Homo oecologicus}

schaftsbürgers von morgen (vgl. Bick \& Meiser, 1977, S. 5). Scherhorn dagegen schreibt ein paar Jahre später (1979, S. 7): „So ist es unverkennbar, daß die Verbrauchererziehung in den letzten Jahren einen beträchtlichen, allerdings auch dringend nötigen Aufschwung nimmt. In allen drei Bereichen - Schule, Erwachsenenbildung, Massenmedien - hat die Intensität zugenommen, mit der Verbrauchererziehung betrieben wird".

Zugenommen hat auch die Handlungsorientiertheit der vermittelten Inhalte. Dennoch muss wohl generell gelten, dass noch immer eine Geringschätzung der ökonomischen Lehrinhalte in der deutschen Bildungspolitik herrschte, und es fehlte an wirtschafts- und sozialwissenschaftlicher Fundierung, an theoretischen Modellen der beteiligten Wissenschaften und damit an einer entsprechenden Lehramtsausbildung.

Wenn man bedenkt, dass die Verbraucherbildung in der hauswirtschaftlich orientierten Mädchenbildung im letzten Drittel des 19. Jahrhunderts ihre Anfänge hatte und Haushalts- sowie Arbeitslehre als „Trägerfächer“ der Verbraucherbildung gelten, ist es umso erstaunlicher, dass in den Veröffentlichungen die Bemühungen in Haushaltslehre und dann auch Haushaltswissenschaft kaum Erwähnung finden. Die Autorin hat sich bereits in den 1970er Jahren der Verbraucherbildung und -erziehung im Zusammenhang von Haushalt, Wirtschaft und Gesellschaft gewidmet und Vorschläge für die schulische Umsetzung unterbreitet (1974, 1976, 1977). Schon zuvor hat Brendl die „Wirtschaftskunde des Haushalts, insbesondere die wirtschaftlichen Aufgaben der Frau und ihre volkswirtschaftliche Verantwortung“ (1954) als Inhalt ausgewiesen, und Kluger hat „Das Verhalten des Konsumenten“ als Problemkreis, der im Rahmen der Arbeitslehre ,anzusprechen“ ist, benannt (1970, S. 104), um nur einige Beispiele zu nennen.

Anfang der 1980er Jahre wurde eine zukunftsorientierte Verbrauchererziehung in der Abkehr von traditionellen Konzepten mit ihren Aufgaben „Preise zu vergleichen“, „preiswert einzukaufen“, „Warenqualität zu überprüfen“ und „Werbemethoden zu durchschauen" hin zu einer Verbrauchererziehung gesehen, die das Kriterium der Umweltverträglichkeit in einem verantwortungsbewussten Konsum berücksichtigt (vgl. Cebulla, 1983). Auch wenn die Bundesregierung bereits 1970 den Umweltschutz zu ihrem Politikfeld machte, 1971 ein erstes Umweltprogramm vorgelegt wurde und auf internationaler Ebene ab 1972 die „Umwelt des Menschen" zum Verhandlungsgegenstand wurde, dauerte es noch ein weiteres Jahrzehnt, bis „Umwelterziehung und -bildung“ in Wissenschaft und Lehramtsausbildung thematisiert wurden und die Kultusministerien - sicher auch aufgrund der KMK-Empfehlungen von 1980 - die Schulen verpflichteten, die Umwelterziehung zu verankern. So hat auch die „Umwelterziehung“ schon ihre Geschichte geschrieben, die mit den jüngsten Forderungen der Bildung für eine nachhaltige Entwicklung wieder einen Höhepunkt erlebt, was hier nicht näher in die Betrachtungen eingehen soll (vgl. vertiefend Fegebank, 1996a und b). Für unsere Überlegungen 


\section{Homo oecologicus |}

ist es entscheidend, dass auch der „Umwelterziehung“ kein eigenes Schulfach gewidmet wird, sondern sie vielmehr integrativer Bestandteil aller Fächer und durchgängiges Prinzip sein soll/te.

Dass das haushälterische Handeln, insbesondere das Konsumverhalten immer auch ein Umweltverhalten ist, war damals in der Haushaltswissenschaft nicht neu und schon in Veröffentlichungen eingegangen. Eine Recherche Mitte der 1980er Jahre ergab, dass in Fachzeitschriften der Haushaltswissenschaft und -lehre mehrfach die Umweltproblematik diskutiert wurde und auch zahlreiche Hinweise für Schule und Unterricht eingebracht wurden (vgl. Fegebank, 1987, S. 353 ff).

Ganz abgesehen von dem umfangreichen Schrifttum und der umfangreichen medialen Präsenz die Umweltprobleme und später auch die Ökologie betreffend, wurde so insbesondere auch das Konsumverhalten als Teil des Umweltverhaltens ins Zentrum gerückt und die Lehrpläne haben entsprechendes aufgenommen, zunächst unter der Zielperspektive des Umweltschutzes. Dennoch musste die Autorin 1999 konstatieren, dass die Aufgabe „Umweltschutz“ - der Schutz vor dem Menschen für den Menschen - noch erhebliche Defizite aufweist (vgl. Fegebank, 1999, S. 13f.). Kritisch war auch anzumerken, dass sich „Umwelterziehung“ - aufgrund von monokausalen Schlussfolgerungen - eher in partiellen Verhaltensmaßregeln gegenüber der „Natur“ erschöpfte, als dass ein Umweltbewusstsein für ökologische Zusammenhänge durch ganzheitliches systemisches Denken angestrebt wurde. Das ist bis heute nicht geleistet worden. Vielmehr wurde das haushälterische Handeln auf das Verbraucherverhalten reduziert, in dem das vom Bundesministerium für Landwirtschaft, Ernährung und Verbraucherschutz geförderte Projekt REVIS, aus dem auch ein Kerncurriculum hervorging eine gewisse Dominanz erreichte und damit wertvolle Ansätze und Themen aus der Haushaltslehre ausblendete.

Verbraucherpolitik und Verbraucherbildung haben einen langen Weg hinter sich, der vorläufig in einem $K M K$-Beschluss von 2013 zur „Verbraucherbildung an Schulen“ endet. Es heißt hier: „Die Verbraucherbildung hat die Entwicklung eines verantwortungsbewussten Verhaltens als Verbraucherinnen und Verbraucher zum Ziel, indem über konsumbezogene Inhalte informiert wird und Kompetenzen im Sinne eines reflektierten und selbstbestimmten Konsumverhaltens erworben werden. Dabei geht es vor allem um den Aufbau einer Haltung, die erworbenen Kompetenzen im Zusammenhang mit Konsumentscheidungen als mündige Verbraucherinnen und Verbraucher heranzuziehen und zu nutzen" (KMK, 2013, S. 2). Dabei folgt Verbraucherbildung durchgängig der Bildung für eine nachhaltige Entwicklung. Die Verbraucherbildung - so heißt es weiter - „weist zahlreiche Anknüpfungspunkte zu bereits etablierten Schulfächern und Lerninhalten auf und ermöglicht in besonderer Weise handlungsorientiertes, interdisziplinäres und vernetztes Lernen. Dieses Lernen soll durch Einbindung außerunterrichtlicher Aktivitäten bzw. außerschulischer Lernorte ergänzt werden" (KMK, 2013, S. 3). Als lebenslanger Prozess und zentrales Element einer Bildung soll Verbraucherbildung ,so- 


\section{Homo oecologicus}

wohl auf aktuelle als auch künftige Herausforderungen im Privat- wie auch im Berufsleben vorbereiten“" (KMK, 2013, S. 2).

\section{Wandel der Leitbilder vom Homo oeconomicus zum Homo oecologicus}

In der rückblickenden Auseinandersetzung mit der Verbraucherpolitik und Verbraucherbildung musste stark selektiv und exemplarisch vorgegangen werden, wobei der Fokus so gelegt wurde, dass zum Ausdruck kam, inwieweit und inwiefern wirtschaftlicher und gesellschaftlicher Wandel stets ein Umdenken in Wissenschaft und Lehre bedingten, die Verbraucher- und Bildungspolitik bestimmten und alle letztlich den Einzelnen in seinem Verhalten zu steuern suchten. Daran hat sich nichts geändert, ebenso wenig wie daran, dass erkannt werden muss, dass Bildung und Erziehung - trotz aller Bemühungen - nicht den erwarteten Erfolg zeitigen. So wird ständig nach „Neuem“ gesucht, werden neue Leitbilder und Wünsche formuliert.

Bevor ausgewählte Leitbilder aus Vergangenheit und Gegenwart, von denen einige schon genannt wurden, mit den dahinterstehenden Intensionen erörtert werden, ist grundsätzlich die Frage nach der Bedeutung von Leitbildern zu stellen und zu klären. Dabei wird sich - in der gebotenen Kürze - an den Ausführungen in der BDI-Studie orientiert. „Leitbilder können einerseits den gegenwärtigen Zustand des Verbraucherverhaltens beschreiben, andererseits dienen sie auch zur Beschreibung eines erwünschten oder als realisierbar eingeschätzten Zustandes in der Zukunft“ (BDI, 2014, S. 19). Mit Leitbildern sind auch „Wünsche“ verbunden, Wünsche als Begehren nach einer Sache oder Fähigkeit, als ein Streben oder zumindest die Hoffnung auf eine Veränderung der Realität oder Wahrnehmung oder das Erreichen eines Zieles für sich selbst oder für einen Anderen. Als Ziel oder Idealzustand werden auf das Erreichen von Leitbildern alle Schutz- und Förderungsmaßnahmen oder Politiken ausgerichtet. Allerdings wird der Begriff des Verbraucherleitbildes, in gesellschafts- und wirtschaftspolitischen und wissenschaftlichen Auseinandersetzungen, aber auch bei juristischen Fragestellungen in jeweils eigenen Zusammenhängen verwendet. Je nachdem, welche Position eingenommen wird, hat sie unmittelbare Wirkung auf das Maß sowie die Art und Weise politischer Maßnahmen“ (BDI, 2014, S. 19). Damit werden an Verbraucher Erwartungen in Form eines normativen Konsumverhaltens gestellt, das Verbraucherbildung und -erziehung bestimmt.

$\mathrm{Zu}$ Zeiten der klassischen Wettbewerbspolitik galt der Verbraucher als Homo oeconomicus, der lange die Überlegungen zu verbraucherpolitischen Maßnahmen bestimmte. Man ging davon aus, dass die Verbraucher mit ihren rationalen Kaufentscheidungen entsprechend ihrer individuellen Präferenzordnung die Produktions- und Anbieterentscheidungen steuern, Nutzenmaximierung anstreben, über 


\section{Homo oecologicus |}

eine vollständige, vollkommene Markttransparenz verfügen und damit eine prinzipielle Konsumentensouveränität gegeben ist, welche auch ein Leitbild aus der Ökonomik ist und den Konsumenten als vollständig informiertes und rational handelndes Wirtschaftssubjekt versteht.

Wegen ihres weitgehend fehlenden Informationsgehalts sind die Annahmen des Homo oeconomicus-Modells in jüngerer Zeit zunehmend kritisiert und durch ein realistischeres Bild vom wirtschaftenden Menschen zu ersetzen versucht worden.

Neben diesen beiden Konzeptionen, deren Relevanz im Verständnis von Marktmechanismen liegt, hat sich mit der Konsumfreiheit ein drittes Leitbild etabliert. „In ökonomischen Untersuchungen wird Konsumfreiheit (meist als uneingeschränkte Wahlfreiheit der Konsumenten) in der Regel diskussionslos und als selbstverständlich vorausgesetzt" (Meyer-Dohm, 1965, S. 23) und es wird gefordert, dass die Konsumenten durch ihre Kaufentscheidungen die Herstellung und Verteilung aller Waren und Dienstleistungen lenken sollen. Obwohl die Freiheitsrechte der Individuen unumstritten sind, ist doch die Konsumfreiheit fragwürdig geworden.

Die Forderung nach dem „mündigen Verbraucher" wird schon lange erhoben, und ist noch immer aktuell, wobei heute häufig im Zusammenhang mit dem mündigen Verbraucher auch die Forderungen nach dem kritischen und/oder verantwortungsbewussten Verbraucher verbunden werden. Es werden mithin immer mehr unterschiedliche Sichtweisen diskutiert.

Zunächst ist festzuhalten, dass Mündigkeit im Sinne von Kompetenz, Informiertheit und Entscheidungsautonomie eine zentrale Annahme herrschender wissenschaftlicher Erklärungsmodelle ist. „Der wirtschaftswissenschaftlichen Deutung liegt bis heute das sogenannte "Informationsmodell' zu Grunde" (BDI, 2014, S. 21). Die Verbraucher werden als weitgehend autonome Individuen gesehen, ,die selbstbestimmt handeln wollen, sollen und können. Sofern sie über die entscheidungsrelevanten Informationen verfügen, sind sie in der Lage, das Marktangebot ,souverän', ihren Präferenzen entsprechend, zu steuern. Das Informationsmodell geht jedoch auch davon aus, dass den Verbrauchern nicht alle Informationen unmittelbar sowie kostenlos zur Verfügung stehen und verständlich sind. Daraus leitet sich die Aufforderung ab, Verbrauchern die notwendigen Informationen zur Verfügung zu stellen. Dann könnten sie eigenständig und rational entscheiden“ (BDI, 2014, S. 21). So wurde der „mündige“ Verbraucher das Ziel aller Maßnahmen der Verbraucherpolitik. Hinzu kommt, dem dann bereits mündigen und selbstbestimmten Verbraucher einen verlässlichen Rechtsrahmen zu geben und für ausreichende Informationsmöglichkeiten zu sorgen (vgl. BDI, 2014, S.30). In diese Richtung der informierten, autonomen, verantwortungsbewussten und kritischen Verbraucher geht so das Leitbild - mit gewissen Interpretationsspielräumen - der politischen Parteien in Deutschland, während in Europa im Leitbild des Europäi- 


\section{Homo oecologicus}

schen Gerichtshofes das juristische, möglichst realitätsnahe Verbraucher-Leitbild wiedergegeben wird, das letztlich aber auch alle Merkmale eines mündigen Verbrauchers aufweist (vgl. BDI, 2014, S. 35).

Nicht zuletzt ist der „mündige Verbraucher“ auch in die Kritik geraten. Die Kritik kommt aus verschiedenen Disziplinen, sie ist mal theoretisch, mal empirisch begründet. Sie lässt sich grob wie folgt zusammenfassen: Verbraucher treffen ihre Entscheidungen nicht alleine, sie konsumieren nicht nur, sondern produzieren auch, ihre Rationalität ist häufig eingeschränkt, und nicht selten sind Normen, Gewohnheiten oder Emotionen der Anstoß für Entscheidungen.

Da kann es nachdenklich machen, dass das Leitbild des „mündigen Verbrauchers" fast alle verbraucherpolitischen Passagen in den Parteiprogrammen dominiert und damit auch die Bildungspolitik bestimmt.

Allerdings gibt es auch eine Richtungsänderung in der Verbraucherpolitik durch den vorsorgenden Verbraucherschutz. „Vorsorgender Verbraucherschutz bedeutet also für alle Akteure die Übernahme von Verantwortung für die Bewahrung bzw. Wiederherstellung einer intakten Um- und Mitwelt im unmittelbar privaten und im gesellschaftlichen Sinne. Dabei ist das Handeln der privaten Haushalte immer auch beeinflusst durch politische und wirtschaftliche Rahmenbedingungen, die fördernd oder einschränkend wirken können, umgekehrt kann das Verhalten der Verbraucher rückwirken auf Politik und anbietende Wirtschaft" (Müller \& Mackert, 2003, S. 21). Hinter diesen Aussagen steht das aktuelle Leitbild des ,nachhaltigen Konsums".

Übergeordnet und schon länger existent ist das Leitbild des Homo oecologicus, das im Laufe seiner kurzen Geschichte stetig verändert und angepasst wurde und damit unterschiedliche Charakterisierungen erfahren hat. Im Gegensatz zum Homo oeconomicus steht Homo oecologicus für ein idealtypisches Menschenbild des perfekt ökologisch denkenden und handelnden Menschen. Dabei ist Ökologie nicht mit Umweltwissenschaft und ökologisch nicht mit umweltgerecht bzw. umweltverträglich gleichzusetzen. Angesichts der seit den 1960 Jahren in der Öffentlichkeit diskutierten Umweltprobleme hat ein gewisser Werte- und Bewusstseinswandel stattgefunden, der in der Ökologiebewegung durch die „ökologische Philosophie“ auf ein höheres Niveau gebracht wurde und mit der Kritik an Technik und Zivilisation eine "neue Ethik" hervorbrachte. Sie sollte fernerhin Basis des Umweltbewusstseins und der Handlungsumsetzung sein. Im Homo oecologicus finden sich so die Forderungen der Umweltbewegung seit den 1980er Jahren nach „,neuen Werten“ und „neuer Moral“, ja nach einem „neuen Menschenbild“ wieder. Das geht weit über den „Menschen als Konsumenten“ hinaus, schließt ihn aber auch ein.

Schon bevor „Homo oecologicus“ zum Begriff wurde, sind - wie bereits erwähnt - Forderungen eines ,umweltgerechten“ bzw. „umweltverträglichen“ Verhaltens in die Umwelterziehung als Teil der Verbraucherbildung eingegangen. 


\section{Homo oecologicus |}

Umwelterziehung ist einerseits auf die Entwicklung des Umweltbewusstseins gerichtet, andererseits auf das aktive Umwelthandeln. Zur Umwelterziehung hat die Kultusministerkonferenz bereits 1982 Forderungen aufgestellt ${ }^{3}$.

Setzte sich Umweltbildung als Prinzip in den Schulen durch, wurde ein besonderer Akzent auf die Umweltethik gelegt. Unter „Umweltethik“ wird auch eine Form ökologischer Intelligenz verstanden, die in fortgeschrittenen Ausprägungsstufen die „Interessen der Umwelt“" stärker berücksichtigt und „mit dem Begriff ökointelligenter Konsum wird ein neues Verständnis von Wohlstand bezeichnet, das nicht auf materiellem Besitz fußt, sondern das ressourceneffiziente Nutzen von Gütern (Produkten, Infrastrukturen, Dienstleistungen) in den Vordergrund stellt. Ökologisches Konsumieren bedeutet dann, bei jeder Kaufentscheidung diejenigen Strategien zu wählen, welche die zur Bedürfnisbefriedigung nötigen Dienstleistungen, im Sinne von Funktionseinheiten, mit dem geringsten Verbrauch an Material und Energie zur Verfügung stellen, d. h. die geringsten Stoffströme verursachen“ (Schmidt-Bleek et al., 1997, S. 83).

Die bisher genannten Forderungen, die das Leitbild des Homo oecologicus prägten und prägen, wurden begleitet von der Forderung nach Nachhaltigkeit, die inzwischen - obwohl selbst Leitbild - zum Hauptmerkmal des Leitbildes Homo oecologicus wurde, wobei es hauptsächlich darum geht, das Konzept der nachhaltigen Entwicklung, das eine zukunftsfähige Politik ermöglichen soll, mitzutragen. Der Begriff „Nachhaltiger Konsum “ wurde in der Agenda 21 auf der RioKonferenz 1992 eingeführt und hat bereits zu einem umfangreichen Schrifttum geführt, auf das hier nur verwiesen werden kann. Beim Leitbild ,nachhaltiger Konsum“ ist interessant, „dass die gängige Definition der nachhaltigen Entwicklung, die auf Bedürfnisbefriedigung fokussiert, letztlich sehr konsumnah angelegt ist. Unter Konsum verstehen wir den Versuch, mit Hilfe marktvermittelter Güter und Dienstleistungen in den Phasen Kauf, Nutzung und Entsorgung die Befriedigung von Bedürfnissen zu erreichen. Auf Basis dieser Definition gibt es in modernen Volkwirtschaften kaum Tätigkeiten, die nicht zugleich auch Konsum darstellen... Bei der nachhaltigen Entwicklung geht es also nicht darum, Konsum abzuschaffen, sondern um dessen umwelt-, sozial- und individualverträgliche Gestaltung“ (Verbraucherpolitik für nachhaltigen Konsum, 2013, S. 4). Nachhaltiger Konsum heißt dann, ,heute so zu konsumieren, dass die Bedürfnisbefriedigung heutiger und zukünftiger Generationen unter Beachtung der Belastbarkeitsgrenzen der Erde nicht gefährdet wird" (BMUB, 2016, S. 1). Für den nachhaltigen Konsum sind von der Bundesregierung relevante Handlungsfelder, wie z. B. „Bildung“, benannt worden, die einen Weg, wie der notwendige Strukturwandel in Wirtschaft und Gesellschaft in Richtung Nachhaltigkeit in Deutschland weiter vorangetrieben werden soll, aufzeigen soll (vgl. BMUB, 2016, S. 1).

Den Handlungsfeldern sind Bedürfnisfelder - wie „Ernährung“ und „Haushalt und Wohnen" - untergeordnet und dazu konkrete Maßnahmen ausgewiesen, die in 


\section{Homo oecologicus}

den verschiedensten Bereichen umzusetzen sind. Eine größere Bedeutung hat der Konsum wohl bisher in Wirtschaft und Gesellschaft kaum erfahren. Mit der Bedeutung wird auch die Frage der Verantwortung aufgeworfen. Konsumenten können die Verantwortung nicht allein tragen, sie agieren in gegebenen Strukturen und befinden sich in gegenseitiger Abhängigkeit mit zahlreichen weiteren Akteuren, so dass das Prinzip der geteilten Verantwortung für einen nachhaltigen Konsum zum Tragen kommt. Danach gibt es keine Hauptverantwortlichen, sondern ein Wechselspiel gegenseitiger Verantwortlichkeiten zahlreicher Akteure. Das bedeutet, dass die Anforderungen an einen nachhaltigen Konsum, insgesamt eine nachhaltige Alltagsgestaltung nicht allein durch „Selbstbestimmung“ und „viel Wissen“, durch die Fähigkeit es einzelnen, Probleme zu lösen und Entscheidungen zu treffen und was sonst noch als Zielperspektiven in Bildung, Beratung, Information und Aufklärung ausgewiesen ist, erreicht wird/werden kann.

Insgesamt ist ein sog. ökologischer Umbau auf globaler Ebene und dann auf den untergeordneten Ebenen erforderlich. An ihm wird bereits gearbeitet, aber er weist noch sehr viele Baustellen auf.

\section{Wunsch und Wirklichkeit}

Wünsche werden hinsichtlich eines „globalen ökologischen Umbaus“ von den verschiedensten Seiten geäußert, wobei hier auf die Bildungsbemühungen, welche auf die Veränderung des Konsumverhaltens und der Alltagsgestaltung im Sinne des Homo oecologicus und implizit des nachhaltigen Konsums ausgerichtet sind, fokussiert wird.

Zusammenfassend kann man den Wunschverbraucher Homo oecologicus als einen Konsumenten bezeichnen, der sich während des gesamten Konsumprozesses - vom Bedürfnis bis zur Bedürfnisbefriedigung - nicht nur Gedanken über die Beziehungen seines Verhaltens zur Umwelt macht, sondern auch die Auswirkungen seines Verhaltens erkennt und letztlich sein Verhalten so ausrichtet, dass er nicht nur seine Gesundheit, sondern auch die Umwelt schützt, indem er sie so wenig wie möglich belastet. Sein Verhalten sollte dabei verantwortungsbewusst, kritisch und von dem Gedanken an Nachhaltigkeit bestimmt sein, sein Konsum sollte „öko-intelligent" sein.

Der Verwirklichung des Leitbildes bzw. dem Weg vom Wunsch zum Ziel steht die Realität deutscher Lebensstile und Verhaltensgewohnheiten gegenüber, wie zahlreiche Studien zum Umwelt- und Konsumverhalten bestätigen, aber auch die „Intelligenz" des Einzelnen. Es wird mehrfach betont bzw. eingeräumt, dass eine grundlegende ,pro-ökologische“ Verhaltensänderung mit einem höheren Bildungsniveau verbunden ist und das in der Folge eine ökologische Gestaltungskompetenz mit sich bringt (vgl. Höfling \& Tretter, 2011, S. 3/4). Zudem sind eine hohe 


\section{Homo oecologicus |}

intellektuelle Kompetenz und eine ständige kritische Reflexion des eigenen Verhaltens nötig.

Die BDI-Studie weist aus: „Die Verbraucherrealität ist ...wesentlich komplizierter, als uns dies das traditionelle ökonomische Verständnis oder die wirtschaftspolitischen Leitlinien vermitteln wollen. Die Entscheidungen der Konsumenten sind weder vollkommen rational, noch sind das Angebot und die sozialen Umstände für die Verbraucher in allen Entscheidungsfällen transparent" (BDI, 2014, S. 4). Auch ist für den Konsumenten nur der subjektiv empfundene Informationsbedarf von Bedeutung.

Verbraucherstudien zeigen zudem ,einen vielschichtigen Verbraucher, der seine Kaufentscheidungen nicht immer vernunftgemäß und aufmerksam trifft, sondern durchaus mit unterschiedlicher Sorgfalt. Die Bandbreite reicht von der intensiv vorbereiteten Kaufentscheidung auf gründlicher Informations- und Abwägungsgrundlage bis zum sog. Spontankauf, zu dem sich der Konsument erst am Verkaufsort entschließt" (BDI, 2014, S. 8).

Lebensweltenmodelle, Milieustudien und Ähnliches versuchen mittlerweile die Realität des Konsumentenverhaltens vollständig zu erfassen, aber „Individualisierung, Erweiterung des Optionenraumes (Multioptionalität) und situationsbedingte Faktoren tragen dazu bei, dass sich die Milieus nicht nur in immer kleinere Untergruppen aufteilen, sondern Individuen zwischen diesen Untergruppen wechseln und gleichzeitig mehreren angehören. Individuelle und sehr differenzierte Beweggründe (Motive, Einstellungen) liegen diesem beobachteten Entscheidungsverhalten der Verbraucher zugrunde. Daraus einen allgemeingültigen verbraucherpolitischen Handlungsbedarf abzuleiten, ist sehr ambitioniert" (BDI, 2014, S. 8). Ebenso ambitioniert sind die ausgewiesenen Kompetenzen, die in der Verbraucher- und Umweltbildung für alle erreicht werden sollen, um dem Leitbild eines Homo oecologicus zu entsprechen.

Das ist sicher ohne einen gesellschaftlichen Wertewandel, der ja ein permanenter ist, nicht möglich. Werte, wie Umweltgerechtigkeit, Askese, soziales Verantwortungsbewusstsein und Nachhaltigkeit, sind dabei dann nicht nur gruppenspezifisch zu vertreten, sondern müssten universell sein. Das bedeutet auch, dass nicht nur der Verbraucher „lernen und handeln“ muss, sondern Wirtschaft und Gesellschaft die Bedingungen schaffen müssen, die ein derart gezieltes Handeln ermöglichen. Solange unser Konsum-Denken von Bequemlichkeit, Fun, Mode und Prestige, Billigpreisen und anderes mehr bestimmt wird, haben "Nachhaltigkeit“ und „Umweltverträglichkeit“ keine großen Chancen. Wenn also eine konkrete Maßnahme (,mehr Wissen“), eine Regel, eine Forderung auf unterschiedliche Lebensstile mit komplexen Handlungsmustern trifft, wird hier kaum ein Erfolg zu verzeichnen sein. So stellt sich die Frage, ob es überhaupt möglich ist, von einem Wunsch zum Ziel zu kommen und dann wie dies vonstattengehen soll. 


\section{Homo oecologicus}

\section{Literatur}

Beer, U. (1967). Konsumerziehung gegen den Konsumzwang. Tübingen: Katzmann.

BDI (Bundesverband der deutschen Industrie e.V.) (Hrsg.) (2014). Studie: Verbraucherleitbild und Positionsbestimmung zum ,, Mündigen Verbraucher “. Berlin: Industrie-Förderung $\mathrm{GmbH}$.

Bick, A. \& Meiser, B. (1977). Taschenbuch der Verbrauchererziehung. Baltmannsweiler: Schneider Verlag Hohengehren.

BMUB (Bundesministerium für Umwelt, Naturschutz, Bau und Reaktorsicherheit) (2016). Nationales Programm für nachhaltigen Konsum.

www.bmub.bund.de/fileadmin/Daten_BMU/Download_PDF/Produkte_und_U mwelt/nat_programm_konsum_bf.pdf

Brendl, M. (1954). Methodik des hauswirtschaftlichen Unterrichts. München: Kösel.

BMWF (Bundesministerium für Wirtschaft und Finanzen) (Hrsg.). (1971). Bericht der Bundesregierung zur Verbraucherpolitik. Frankfurt/Main: UnionDruckerei und Verlagsanstalt GmbH.

BMWF (Bundesministerium für Wirtschaft und Finanzen) (Hrsg.) (1972). Es geht um den Verbraucher. Ein Jahr Verbraucherbericht. Köln: Gebr. Nettesheim oHG.

Cebulla, E. (1983). Grundlegende Gedanken einer zukunftsorientierten Verbrauchererziehung im Kindergarten. In R. Rock \& K.-H. Schaffartzik (Hrsg.), Verbraucherarbeit: Herausforderungen an die Zukunft (S. 229-240). Frankfurt/Main: Campus.

Fegebank, B. (1974). Erziehung zum Verbrauch? Die berufsbildende Schule, H. 3, 171-179.

Fegebank, B. (1976). Das Verbraucherverhalten als Gegenstand von Unterricht und Erwachsenenbildung. Die berufsbildende Schule, H. 3, 151-158.

Fegebank, B. (1977). Verbrauchererziehung in der Schule. Hauswirtschaftliche Bildung, H. 3, S. 113-117.

Fegebank, B. (1987). Umwelterziehung im Rahmen der Bildung des Menschen für den Haushalt (Hauswirtschaft/Haushaltlehre). In R. Lob \& V. Wichert (Hrsg.), Schulische Umwelterziehung außerhalb der Naturwissenschaften (S. 353-392). Frankfurt/Main: Peter Lang.

Fegebank, B. (1999). Haushaltshandeln und Umweltverhalten. In B. Fegebank, U. Müller \& B. Schramm (Hrsg.), Haushaltshandeln ist Umweltverhalten! (S. 5-14). TU Dresden: Selbstverlag.

Fegebank, B. (1999a). Umweltgerechter Einkauf. In B. Fegebank, U. Müller \& B. Schramm (Hrsg.), Haushaltshandeln ist Umweltverhalten! (S. 43-50). TU Dresden: Selbstverlag. 


\section{Homo oecologicus |}

Fegebank, B. (1999b). Umweltbildung - Umwelterziehung. In B. Fegebank, U. Müller \& B. Schramm (Hrsg.), Haushaltshandeln ist Umweltverhalten! (S. 157-168). TU Dresden: Selbstverlag.

Fegebank, B. (2002). Der Homo oecologicus oder: Umweltverträgliches Verbraucherverhalten. In B. Fegebank, U. Müller \& B. Schramm (Hrsg.), Verbraucher und Markt (S. 31-42). Dresden: Selbstverlag.

Fegebank, B. (2016a). „Nachhaltigkeit“ zwischen Idealität und Realität (Teil 1). Hauswirtschaft und Wissenschaft, 64(1), S. 33-39.

Fegebank, B. (2016b). „Nachhaltigkeit“" zwischen Idealität und Realität (Teil 2). Hauswirtschaft und Wissenschaft, 64(2), S. 63-68.

Galbraith, J. K. (1959). Gesellschaft im Überfluß. München: Droemer Knaur.

Hasselberg, P. v. \& Heintz, S. (1971). Vorwort. In T. Veblen, Theorie der feinen Leute (S. 7-15), München: dtv.

Höfling, S. \& Tretter, F. (2011). Homo oecologicus: Menschenbild für die nachhaltige Entwicklung im 21. Jahrhundert-Philosophische Aspekte (Tagungsbericht).

http://www.hss.de/fileadmin/media/downloads/Publikationen/121213_AMZ82_Internetversion.pdf

Katona, G. (1965). Der Massenkonsum. Eine Psychologie der neuen Käuferschichten. Düsseldorf: Econ.

KMK (Kultusministerkonferenz) (2013). Verbraucherbildung an Schulen (Beschluss der Kultusministerkonferenz vom 12.09.2013).

http://www.kmk.org/fileadmin/Dateien/veroeffentlichungen_beschluesse/2013/ 2013_09_12-Verbraucherbildung.pdf

Kluger, I. (1970). Hauswirtschaftsunterricht in der Hauptschule. Wuppertal: A. Henn Verlag.

Müller, E. \& Mackert, H. (2003). Bildung für den Haushalt und Konsum als vorsorgender Verbraucherschutz. Politik und Zeitgeschichte, B 9, S. 20-26.

Packard, V. (1957). Die geheimen Verführer. Düsseldorf: Econ.

Scherhorn, G. (1975). Verbraucherinteresse und Verbraucherpolitik. Göttingen: Otto Schwartz \& Co.

Scherhorn, G. (Hrsg.) (1979). Verbrauchererziehung in der Bundesrepublik Deutschland. Baltmannsweiler: Schneider Verlag Hohengehren.

Schmidt-Bleek, F., Merten, T. \& Tischner, U. (Hrsg.) (1997). Ökointelligentes Produzieren und Konsumieren. Berlin: Birkhäuser Verlag. http://dx.doi.org/10.1007/978-3-0348-6104-5

Schmölders, G. (1962). Geschichte der Volkswirtschaftslehre. Reinbek bei Hamburg: Rowohlt.

Schmucker, H. (1980). Studien zur empirischen Haushalts- und Verbrauchsforschung. Berlin: Duncker \& Humblot. 


\section{Homo oecologicus}

Solomon, M., Bamossy, G. \& Askegaard, S. (2001). Konsumentenverhalten. Der europäische Markt. München: Pearson Studium.

Veblen, T. (1971). Theorie der feinen Leute. München: dtv.

Verbraucherpolitik für nachhaltigen Konsum (2013). Verbraucherpolitische Perspektiven für eine nachhaltige Transformation von Wirtschaft und Gesellschaft. https:/www.aloenk.tu-berlin.de/fileadmin/fg165/Aktuelles/

Stellungnahme_Nachhaltiger_Konsum_-_final.pdf

\section{Anmerkungen}

1 Der Begriff „Verbraucher“ steht für alle Menschen gleichermaßen und wird daher nicht in eine weibliche und männliche Form gesplittet.

2 Im Laufe der Geschichte und heute in den einzelnen Bundesländern existieren wechselnde Termini für die Schulfächer. Als bisher, neutralster Begriff einigte sich die ehemalige Bundesfachgruppe auf Haushaltslehre.

3 Dieser Beschluss, der eine Art Grundsatzempfehlung für die zuständigen Bundesländer war, ersetzte den bis dahin gültigen der $K M K$ vom 30.9.1953 über Naturschutz und Landschaftspflege sowie Tierschutz. Die Umsetzung des Beschlusses von 1980 erfolgte $z$. T. erheblich später.

\section{Verfasserin}

Prof. $^{\text {in }}$ Dr. Dr. Barbara Fegebank

Seniorprofessorin

Technische Universität Dresden

Fakultät Erziehungswissenschaften

Institut für Berufspädagogik und Berufliche Didaktiken

D-01062 Dresden

E-Mail: Barbara.Fegebank@tu-dresden.de 